\title{
Compact, Non-invasive Frequency Domain Lifetime Differentiation of Collagens and Elastin
}

\author{
Rui Liu ${ }^{1 *}$, Zhengtuo Zhao ${ }^{1 *}$, Luwei Zou ${ }^{1}$, Qiyin Fang ${ }^{2}$, Lin Chen ${ }^{3}$, Alan Argento ${ }^{1}$, Joe F. Lo ${ }^{1 * *}$ \\ ${ }^{1}$ Department of Mechanical Engineering, University of Michigan-Dearborn, Dearborn, Michigan, USA \\ ${ }^{2}$ Department of Engineering Physics, McMaster University, Hamilton, ON, Canada \\ ${ }^{3}$ Center for Wound Repair \& Regeneration, University of Illinois-Chicago, Chicago, IL, USA \\ *equal contribution
}

**Corresponding Author:

Joe Fujiou Lo

Bioengineering Program

Department of Mechanical Engineering

University of Michigan-Dearborn

HPEC 1332

4901 Evergreen Rd.

Dearborn MI 48128

jfjlo@umich.edu

(313) 593-0913 


\begin{abstract}
:
Changes in the composition of type I and type III collagen in tissue can shed light on various diseases. However, many of the current collagen detection techniques require invasive and destructive tissue sampling. In this study, a low cost, low complexity light emitting diode (LED) based system was developed to realize both non-invasive detection and specific discrimination of collagen and elastin variations in tissue based on fluorescence lifetimes. Modulated LED excitation was applied to frequency domain (FD) fluorescence lifetime spectroscopy to calculate tissue autofluorescence lifetimes. Using this method, fluorescence lifetimes from collagen type I versus type III were clearly separated at 3.95 ns and $5.01 \mathrm{~ns}$, respectively, distinct from the elastin lifetime at $6.78 \mathrm{~ns}$. The probe was tested on bovine ocular tissues, with cornea showing much shorter average lifetime of 4.27 ns than sclera at 7.48 ns. Furthermore, measurements of an $8 \mathrm{~mm}$ murine skin wound at 14 days post-wounding also showed distinct, longer average lifetimes at 9.74 ns versus normal skin at 6.72 ns. This FD tissue detection technique can potentially offer a way to examine tissue structures and discern the underlying pathology nondestructively.
\end{abstract}

Keywords: collagen I, collagen III, elastin, frequency domain, fluorescence lifetime, extra cellular matrix 


\section{Introduction:}

Collagen is the main load-bearing structural protein in the extra cellular matrix (ECM) of biological tissue. The quantity, type, and orientation of collagen greatly influence the way tissues carry stress, stretch, and maintain integrity. Elastin reinforces the collagen network and provides resilience to tissues. To study the collagen and elastin composition in biological tissues, and their changes in diseases, a noninvasive detection technique is required. In this study, a general method is developed to detect and differentiate ECM proteins. Here, detections were demonstrated with type I collagen, type III collagen and elastin in the context of their measurements in skin wounds and ocular tissues [1-3]. The method uses a low cost, low complexity light emitting diode (LED) system to realize both non-invasive detection and specific discrimination of collagen and elastin variations in tissue. Modulated LED excitation was applied to frequency domain (FD) fluorescence lifetime spectroscopy to determine tissue autofluorescence lifetimes, Figure 1. Using the novel LED-based system, fluorescence lifetimes from collagen type I, collagen type III and elastin can be clearly distinguished. These frequency domain-based lifetimes, especially the direct comparison of type I and III collagen, are reported here for the first time, in contrast to time domain measurements in the literature. The extension of the method to detect other collagen types and ECM components is also discussed. Due to the low cost of LED and photodiode components, low complexity of the fiber optic probe, and the possibility of integrating phase and demodulation analyses on an integrated circuit, the developed technique is well suited to portable applications. Applications in implantable sensors, field diagnostics, and clinical monitoring can benefit from this technique to measure collagen and elastin distributions in biological tissues over time. 
A)

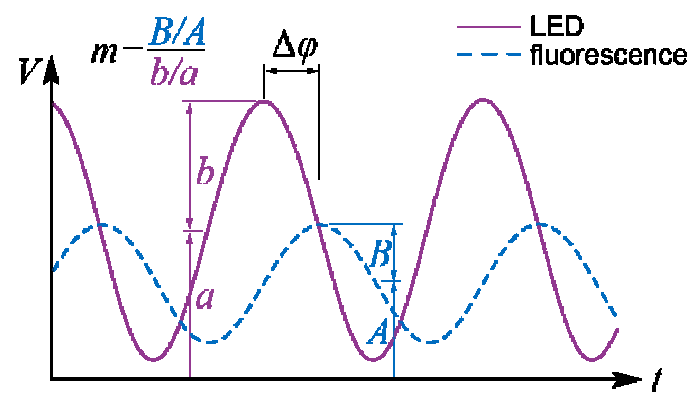

C)

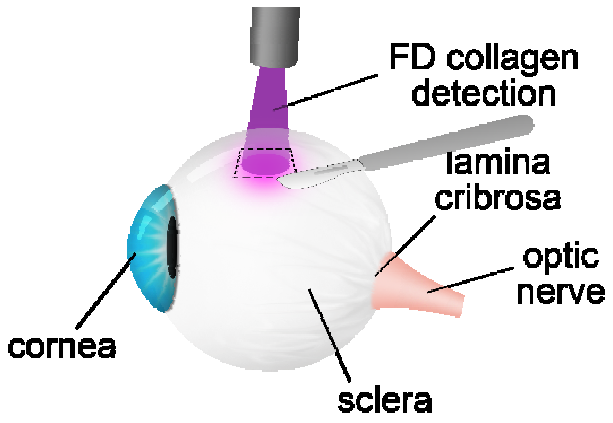

B)

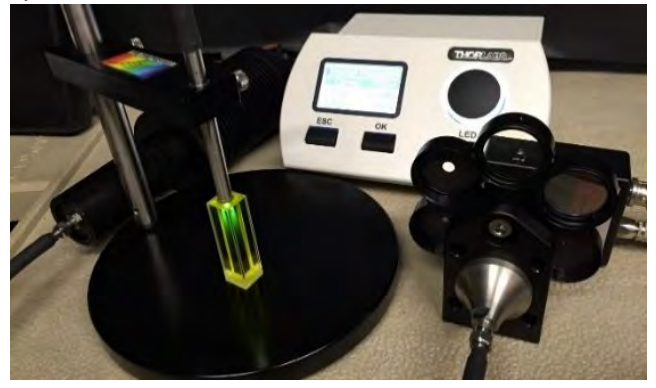

D)



Figure 1. Fiber based frequency domain collagen and elastin detection system. A) Frequency domain fluorescence lifetime is based on the phase shift delay and amplitude demodulation of the fluorescence emission when a sample is excited by a modulated light source. B) The components of the systems are compact and cost-effective. This system can be applied to C) non-invasive probing of ocular tissue, and D) non-contact monitoring of wound healing. (Note that ocular and skin tissues were cut into samples and measured in this study.)

\section{Collagen and ECM Composition of Skin Wounds and Ocular Tissue}

In skin wounds, collagen remodeling occurring underneath a closing wound cannot be easily assessed by visual monitoring. The primary structural component of normal dermis is mostly collagen, with elastin, glycoproteins, and glycosaminoglycans also present. During the proliferation phase, fibroblasts proliferate in the wound and produce ECM, especially collagens, to replace the provisional matrix formed in homeostasis/inflammatory stage of the earlier wound healing process to establish scar tissue [4]. Normal skin and wounds that have undergone collagen remodeling contain more mature crosslinked collagen I fibers, while immature wounds contain more parallel collagen III fibers $[5,6]$. Elastin is another major protein component of the ECM produced by fibroblasts and smooth muscle cells, which provide resilience to the skin and other tissues. It is abnormally expressed during skin wound healing which partially contributes to the impaired breaking strength of scars compared with unwounded skin 
[7]. Restoration of normal ECM architecture is necessary for skin wounds to gain the tensile strength that is required to maintain tissue integrity [1]. Full thickness murine wounds have been used as important models to study wound healing, including collagen remodeling processes.

In the eye, the type and orientation of collagen fibers vary by region and are closely related to the eye's functions $[2,8,9]$. For example, in the peripapillary region where the optic nerve exits the sclera, there is a predominant circumferential fiber alignment [10] that likely functions to control deformation and stress in the optic nerve head tissues. Similar to skin and other connective tissues, sclera is primarily composed of a matrix containing collagens, elastin [11], and proteoglycans [12]. The result is a tissue having strength and viscoelastic properties that help to protect the eye from severe loads, and also from brief elevations in intraocular pressure due to eye movements and other events such as rubbing. In the cornea, the lamellae are composed of collagen fibrils of uniform diameter running parallel to one another; however, in the sclera the collagen fibrils have varying diameter with an irregular branching pattern $[13,14]$. Human eye tissue contains about $90 \%$ type I collagen and less than $5 \%$ type III collagen [2]. Levels of type III collagen in the bovine cornea average less than $1 \%$ in animals older than 3 months $[15,16]$. Disorders in the ocular structure play a role in diseases such as glaucoma $[17,18]$ which manifests as damage to the tissues of the optic nerve head, and the corneal thinning disease keratoconus [19]. Structural problems of the sclera have also been implicated in myopia [20]. Abnormal elastin has been found in the lamina cribrosa tissue, which bridges the opening in the sclera at the optic nerve head, of glaucomatous eyes [20]. A non-invasive assay of tissue would aid research into eye disorders such as these as well, as skin wounds discussed above, and could eventually be used as a diagnostic tool.

\section{Existing ECM Detection Techniques}

Several techniques are currently used to measure tissue ECM compositions, such as histological staining, histological autofluorescence, and non-invasive tissue optical biopsy. Tissue histology has wellestablished analysis and quantification methods in techniques such as immunofluorescence [21, 22] and picrosirius staining microscopy [23, 24]. However, histology requires invasive sampling and destructive sectioning to produce samples for measurement. On the other hand, laser-induced tissue fluorescence and microscopy techniques have been developed for non-invasive tissue monitoring, but have not enabled differentiation of collagen types specifically [25-27]. Laser fluorescence and microscopy instrumentation is also prohibitively costly and complicated for widespread biomedical application. 


\section{Characteristics of Collagen and Tissue Autofluorescence}

Autofluorescence from collagen (i.e. without staining) is commonly observed in cell and tissue microscopy. The major collagen fluorophores are lysine derived pyridinium, tyrosine, and phenylalanine groups [28-31], which can be affected by crosslinking, glycation, and their compositions in different types of collagens [32-34]. Collagen autofluorescence, in combination with other endogenous fluorophores, can provide differentiation between normal and cancerous tissues [35, 36], promising tumor demarcation in minimally invasive surgeries [37, 38]. Moreover, collagen fiber orientation and crystallinity enable second harmonic generation (SHG) using laser induced autofluorescence [39-43]. Both multiphoton collagen autofluorescence and SHG have been adapted for monitoring skin aging [44, 45] and investigating ocular pathology [46-50]. Despite the ability to detect endogenous fluorophorese.g. $\mathrm{NADH}+$, elastin, and collagen-no differentiation of collagen types using SHG and multiphoton microscopy has been demonstrated. This is because very little differences exist in the spectral domain of collagen types, e.g. I versus III. However, autofluorescences from different collagen types evidently have strong differences in the time domain [51,52]-their lifetimes are significantly different and sensitive to the crosslinking and glycation, as mentioned earlier. Therefore, fluorescence lifetime spectroscopy, either in the time-domain or frequency domain, can provide non-invasive differentiation of collagen types in tissue.

\section{Time and Frequency Domain Methods for Collagen and Elastin Fluorescence Lifetime}

Collagen and elastin autofluorescence, and fluorescence in general, can be modeled as a linear, timeinvariant system with a characteristic impulse response function specific to a particular fluorophore [5355]. Therefore, the input (excitation) and output (emission) of such a system can be considered in time and frequency domains. In the time domain, the input excitation is convolved with the impulse response to yield the emission intensity over time. This time domain method has been demonstrated with subnanosecond pulsed lasers and fast photomultiplier tube (PMT) detectors to reconstruct collagen fluorescence impulse response decays, with associated lifetime constants [56-59]. While the time domain method obtains more information over shorter pulses, the cost of the pulsed lasers, triggering electronics, and PMT detectors, in addition to the complexity of the system, prevent its wider use in biomedical applications. On the other hand, the frequency domain method applies a sinusoidal modulation to the excitation intensity, resulting in a sinusoidal emission with amplitude suppression and 
phase shift, as expected from a linear time-invariant system, Figure 1B. The frequency domain lifetime method is potentially a cost-effective alternative to monitoring biological tissues [60, 61]. Optimum modulation frequencies for tissue fluorophores-e.g. collagen, elastin, $\mathrm{NADH}-$ range from $10-100 \mathrm{MHz}$ [62], lowering the requirements for the LED modulation electronics, photodetector temporal response, as well as signal digitization and recording speed $[63,64]$. Moreover, a single multi-channel oscilloscope can perform the timing and acquisitions for both the excitation and emissions signals. Using the phase $\Delta \phi$ and modulation $m$ from oscilloscope measurements, two lifetimes, $\tau_{\phi}=\omega^{-1} \tan \Delta \phi$ and $\tau_{m}=\omega^{-1}\left(m^{-2}-1\right)^{1 / 2}$, can be calculated and combined via multi-exponential models to find characteristic lifetimes of a sample. In this study, the characteristic lifetimes of fluorescence standards, purified collagen proteins, and ocular and skin tissue samples were measured to demonstrate the system's utility. 


\section{Methods}

\section{FD ECM Detection System Overview}

The system is composed of the following components: A modulated $365 \mathrm{~nm}$ wavelength LED (DC3100365 , Thorlabs, NJ) was used as the light source along with a $275-375 \mathrm{~nm}$ bandpass filter. This sinusoid modulated excitation light was carried by a 7 around 1 UV-resistant fiber probe (Stellarnet, FL) to illuminate the sample at frequencies from $10 \mathrm{MHz}$ to $60 \mathrm{MHz}$. The emission and the reflected/scattered light from the sample were collected by the 7 fibers and the reflected/scattered light was then filtered by two $400 \mathrm{~nm}$ longpass filters. An avalanche photodiode (APD-110C, Thorlabs, NJ) picked up the filtered emission intensity and compared its modulation with the LED source on an oscilloscope, Figure 2. Because of the availability of modulated LED, fast avalanche photodiode, and sub $100 \mathrm{MHz}$ oscilloscope at affordable costs, the FD collagen system in this study does not require extra signal generator or heterodyne electronics $[65,66]$, and has a generally low overall system cost.

\section{System Optomechanics}

To couple the light from the LED, a plano-convex lens was used to collimate the light through the 375 $\mathrm{nm}$ low pass filter and focused by a second plano-convex lens onto the fiber probe. The fiber probe configuration has one illumination core in the center and 7 surrounding collection cores. This gives higher collection efficiency with smaller detection spot provided by the single illumination core. Similar to the LED coupling, detector coupling was achieved by collimating the collection fiber output through longpass filters. Then the signal was focused on the APD with a pair of plano-convex lenses (as shown in Figure 2). Moreover, the longpass filters, as well as neutral density filters, were mounted on a filter wheel to enable them to be moved in and out of the light path during normal and calibration operations. The components details and costs are listed in Table 1. 




* Using Halon reflectance standard at the probe tip.

Figure 2. FD ECM detection optomechanics design. At the modulated LED light source, a pair of planoconvex lenses $(\$ 25.4, \mathrm{f}=25.4)$ help to couple light through an excitation filter (275-375 $\mathrm{nm}$ bandpass) into a $600 \mu \mathrm{m}$ fiber. This excitation is illuminated on a fluorescence sample, and the emission is collected by seven $600 \mu \mathrm{m}$ fibers into the detector coupling block. In the block, a plano-convex lens $(\phi 12.7, \mathrm{f}=20.0)$ collimates the emission intensity through a pair of $400 \mathrm{~nm}$ longpass filters, which can also be replaced by either a pin hole or a neutral density filter during calibration procedures. The filtered emission is then focused by an aspheric lens $(\phi 25.0, \mathrm{f}=20.0)$ onto a fast, avalanche photodiode. The efficiencies of the system can be interpreted by the optical power measured at various positions, as well as typical sample fluorescence from FAD and Collagen I.

Table 1. System Components:

\begin{tabular}{l|l|l}
\hline Component & Relevant Spec & Price \\
\hline Thorlabs Modulated LED Source & $700 \mathrm{~mA}, 10-90 \mathrm{MHz}$ sinusoid & $\$ 2510$ \\
1" Lens tube components & SM1 lens tube, SM1 collimator & $\$ 450$ \\
SM1 optics & 1" lens and lens tubes & $\$ 200$ \\
Colored Glass Bandpass & $275-375 \mathrm{~nm}$ & $\$ 86$ \\
Stellarnet 7 around 1 probe & $600 \mu \mathrm{m}$, solarization resistant & $\$ 600$ \\
Manual filter wheel & 6 position, 1" optics & $\$ 145$ \\
Thorlabs Longpass FEL0400 & $400 \mathrm{~nm}$ & $\$ 73(2)$ \\
Thorlabs APD120A2 Photodiode & DC-50 MHz (3dB), 2.5e6 V/W & $\$ 1070$
\end{tabular}




\section{System Calibration}

Rigol DS1000B Oscilloscope

$200 \mathrm{MHz}, 2 \mathrm{GSa} / \mathrm{s}$

$\$ 945$

Phase shift and modulation depth ratio of the system were calibrated for comparisons between solid and liquid sample measurements. During these calibration measurements, the emission filters were switched out and replaced with neutral density filters or pin holes to further reduce intensity. For the reference phase angles, reflected/scattered light was measured at 10 to $60 \mathrm{MHz}$ from frosted glass, water, and ethanol media to compare with measurements from powder/fiber form, aqueous solutions and ethanol solutions, respectively. For the reference modulation depth ratios, the LED waveform's b/a ratios were measured for the frosted glass, water, and ethanol solutions, to parallel those measured for phase references.

\section{Fluorescence Standard, Purified Protein, and Tissue Sample Preparation}

For comparison to known lifetime standards, three fluorescence standards were measured: flavin adenine dinucleotide (FAD), fluorescein isothiocyanate (FITC), and 9-Anthracenecarboxylic acid (9CA). 0.1 mM FAD was prepared in water while $0.1 \mathrm{mM}$ FITC and 9CA were prepared in ethanol. FAD, FITC, and 9CA have lifetimes of $2.57,3.94$, and $11.75 \mathrm{~ns}$, covering the range of lifetimes expected form the purified ECM proteins. Then, three purified ECM proteins were measured-collagen I, collagen III, and elastin-to represent important ECM components in ocular and skin tissues. Purified proteins were measured in solid powder or fiber forms. Finally, lifetime measurements were conducted in bovine sclera and cornea tissues, and murine normal and wound skin tissues (14 days post-wounding).

In this study, bovine tissue is used because it is plentiful and of similar consistency to human eye tissue and is often used as a precursor to experiments using cadaveric or surgical specimens of human origin $[16,67]$. Bovine eyes were obtained from a local slaughterhouse and tested within 48 hours of the animal's death. Scleral and corneal tissues were dissected from the surrounding fat and extraocular muscles, then separated from the internal contents of the eye. The scleral and corneal tissues were then cut into $5 \mathrm{~mm}$ squares for measurement. Measurements were done normal to the external surface of the tissue samples.

$\mathrm{BALB} / \mathrm{C}$ mice were used in the skin wound model. Murine experimental procedures followed the University of Illinois guidelines for the humane treatment of animals and were approved by the University of Illinois Institutional Animal Care and Use Committee. Wound models were created using 5 $\mathrm{mm}$ punch biopsies on day 0 and collected on day 14 post-wounding with $8 \mathrm{~mm}$ punch biopsies. 
Immediately after biopsy sampling, skin and wound tissues were measured normal to the external surface of the tissues.

\section{Frequency Domain Analysis}

During data acquisition, the raw emission signals were averaged 128 times in the oscilloscope to reduce effects of noise, then the averaged signals were imported into MATLAB (MathWorks, MA) and fitted to sine functions. The least-square method was employed to fit the raw signals to a sine function with known frequencies.

$$
y=a+b \sin (\omega x-\phi), \text { or } y=A+B \sin (\omega x-\phi)
$$

Where $\omega$ is known when the sample is excited under the LED light with a certain frequency, $x$ is the time axle, and $y$ is the raw voltage data. The parameters phase $\phi$, amplitude $b$, offset $a$ for the excitation and phase $\phi$, amplitude $B$, offset $A$ for the emission waveforms (Figure 1A) are readily extracted from the curve fitting. However, there were noticeable DC offsets in the raw signals depending on measurement frequencies and oscilloscope settings. Therefore, DC offsets from dark readings (LED turned off) at specific measurement conditions were subtracted from the raw data before fitting and data processing. This process allowed more accurate calculations of the phase shift angles and modulation depths, without peak uncertainties, amplitude drifts, and waveform asymmetries from one part of the raw data to another, leading to better lifetime measurements.

After getting the phase angles and modulation depth ratios between $10 \mathrm{MHz}$ to $60 \mathrm{MHz}$, the data was fitted to a multi-exponential model as described in [68], where the fluorescence impulse response function is assumed to be:

$$
I(t)=\sum_{i=1}^{n} \alpha_{i} \mathrm{e}^{-t / r_{i}}
$$

Where $\alpha_{i}$ is the lifetime fraction of each component $\tau_{i}$ and $\sum \alpha_{i}=1$. Thus $I(t)$ appears as a decay curve containing multiple exponents represented in the sample. The calculated phase shift angle $\phi_{o w}$ and modulation $m_{\sigma \omega}$ can then be obtained from the sine $N_{\omega}$ and cosine $D_{\omega}$ transformation of $I(t)$ :

$$
\begin{aligned}
& N_{\omega}=\frac{\int_{0}^{\infty} I(t) \sin \omega t d t}{\int_{0}^{\infty} I(t) d t} \\
& D_{\omega}=\frac{\int_{0}^{\infty} I(t) \cos \omega t d t}{\int_{0}^{\infty} I(t) d t}
\end{aligned}
$$


Where their numerical values can be calculated by:

$$
\begin{aligned}
& N_{\omega^{\prime}} J=\sum_{i=1}^{n} \frac{\alpha_{i} \omega \pi_{i}^{n}}{1+\omega^{3} \tau_{i}^{n}} \\
& D_{\omega^{\prime}} J=\sum_{i=1}^{n} \frac{\alpha_{i} \tau_{i}}{1+\omega^{2} z_{i}^{n}}
\end{aligned}
$$

Where $I=\sum \alpha_{i} \tau_{i}$.

Thus the phase shift angle $\phi_{o w}$ and modulation $m_{\sigma \omega}$ can be described by:

$$
\begin{aligned}
& \phi_{\sigma \omega}=\arctan \left(N_{\omega} / D_{\omega}\right) \\
& m_{c \omega}=\left(N_{\omega}^{2}+D_{\omega}^{2}\right)^{1 / 2}
\end{aligned}
$$

An error-weighted $\chi^{2}$ sum of the squares of the deviations between the measured and calculated values is minimized to find the best fitting of multi-exponential model.

$$
\chi^{2}=\Sigma_{\omega} \frac{1}{\sigma_{\phi}^{2}}\left(\phi_{\omega}-\phi_{\omega}\right)^{2}+\Sigma_{\omega} \frac{1}{\sigma_{m}^{2}}\left(m_{\omega}-m_{\sigma \omega}\right)^{2}
$$

Where $\sigma_{\phi}$ and $\sigma_{m}$ are the typical uncertainties in the phase and modulation data, respectively. Respective standard deviations from their measurements were used to represent these uncertainties. A single exponential model was used for fluorescence standard FAD, FITC, and 9CA, to be consistent with other reported studies $[58,64,69]$. A two-exponential model was used for protein and tissue samples, where fractional $\alpha_{1}, \alpha_{2}$, and their respective $\tau_{1}$ and $\tau_{2}$ were calculated by minimizing the $\chi^{2}$ function describing deviations of their $\phi_{\omega}$ and $m_{\omega}$ values.

\section{Data Processing:}

The LED trigger and APD detector signals were acquired using a $200 \mathrm{MHz}$ oscilloscope (Rigol DS1000B). The data was then loaded into Matlab (Mathworks) for analysis. The curve fitting module was used to fit a sinusoid using the data, at pre-defined frequencies corresponding to the LED excitation, e.g. 10, 20 $\mathrm{MHz}$, etc. Curve fitting was chosen over Fourier Transform due to its simple implementation in Matlab, where the amplitude, DC offset, and phase shift can be directly extracted, without ambiguity. No obvious processing time penalties were observed using this method. Future implementation of Fourier Transform, however, can be beneficial in an embedded data processing unit. After extraction of the 
phase and modulation parameters, they were implemented into a minimization function to fit into the appropriate single/multi-exponential model. 


\section{Results and Discussion}

To characterize the FD ECM detection system, the calibration results and fluorescence spectra of each standard, protein, and tissue samples were presented. Then, the FD phase shift and demodulation results were presented. Next, the single and multi-exponential fitted lifetimes were summarized in Tabulated form. Finally, the relationship between temporal resolution and sample detectivity was characterized to describe the sensitivity of the FD system.

\section{System Calibration Results}

The phase angles of mirror/water/ethanol references were fairly close to each other, Figure $\mathbf{3 A}$. As with phase references, modulation references did not change significantly among all samples, Figure 3B.

A)

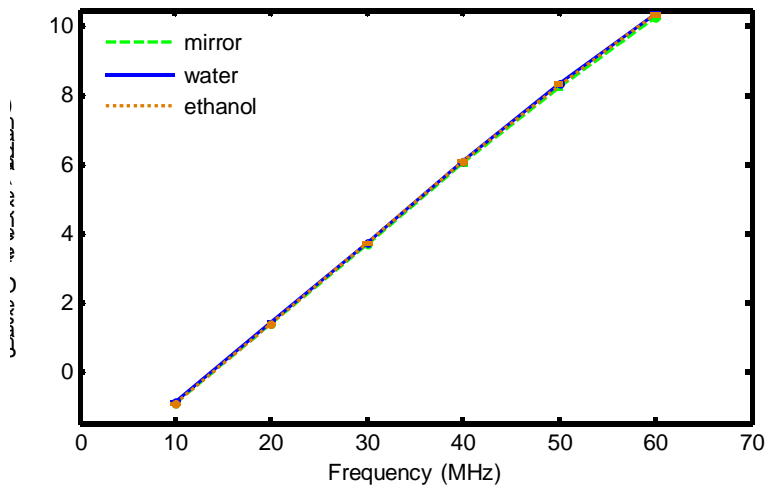

B)

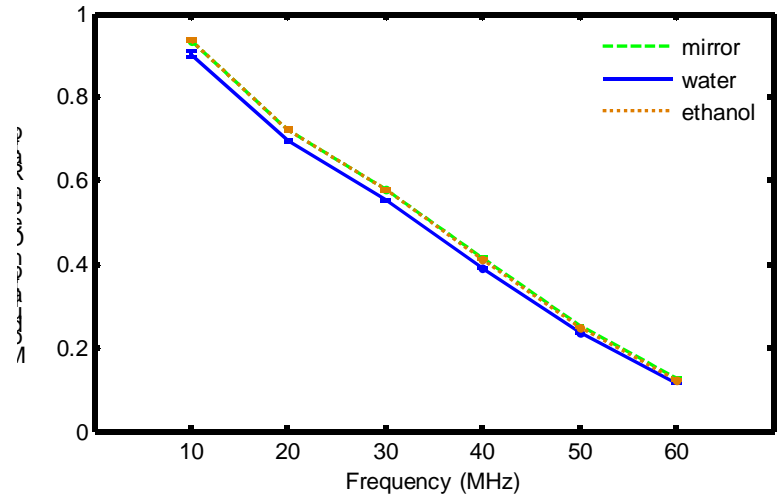

Figure 3. References phase and modulation ratios. A) Phase delays versus frequency of LED modulation.

B) Modulation depth ratios versus frequency of LED modulation. Measurements were made using mirror/water/ethanol references to represent the solid, aqueous, and ethanol solvated samples. Error bar denotes standard deviation, $\mathrm{n}=3$

\section{Spectral Domain Alone Cannot Distinguish Collagen Types and Elastin}

Spectral data showed that 9CA peaked at $450 \mathrm{~nm}$, FITC at $520 \mathrm{~nm}$, and FAD at $530 \mathrm{~nm}$, Figure 4. Protein spectra generally overlapped for collagen I/III and elastin, but showed significant intensity differences above $450 \mathrm{~nm}$. This spectral overlap, combined with other present chromophores like hemoglobin, makes tissue reflectance complicated and difficult to interpret. In the FD collagen system described in this manuscript, lifetime measurements were employed to distinguish purified protein components by integrating all wavelengths above $400 \mathrm{~nm}$. 
A)

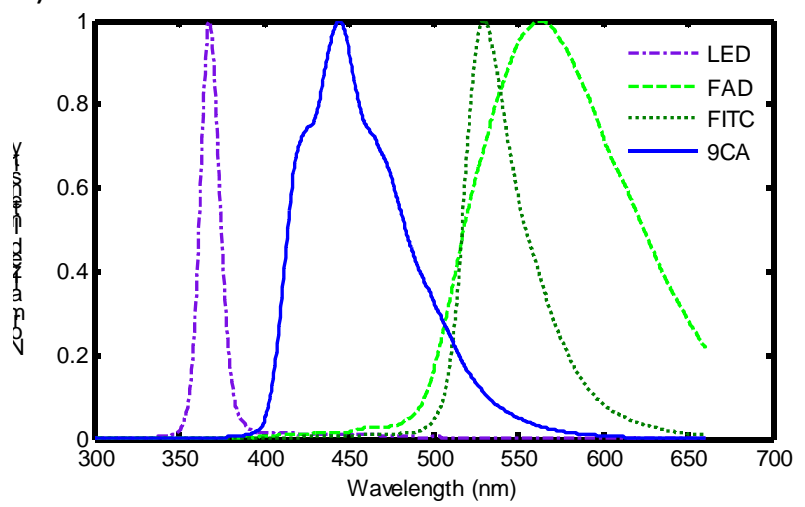

C)

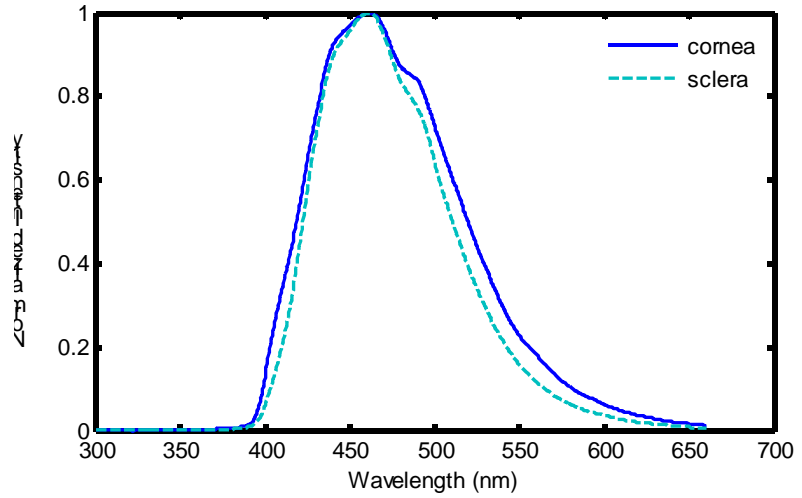

B)

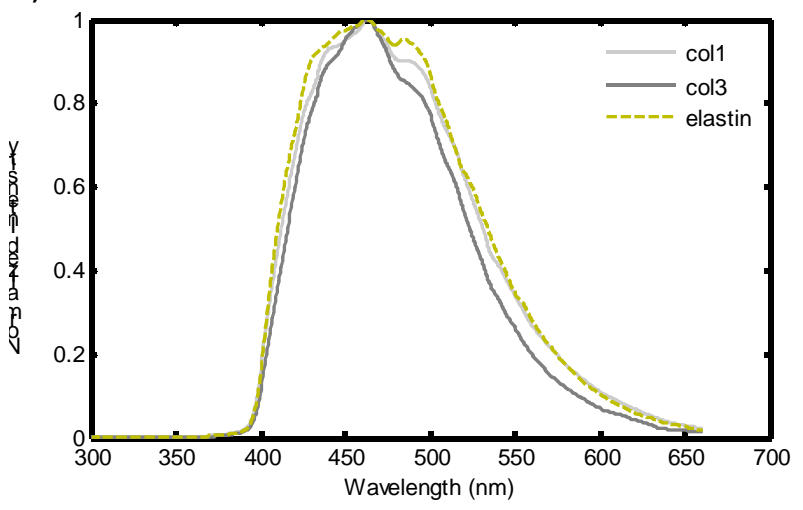

D)

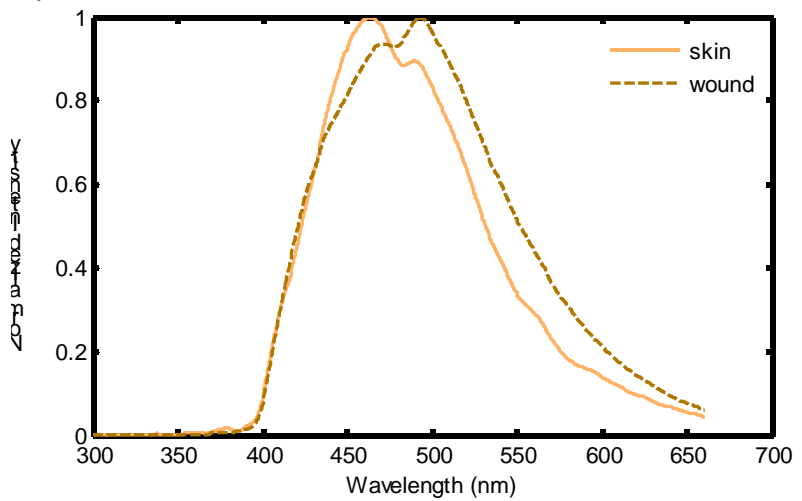

Figure 4. Fluorescence spectra of standards, proteins, and complex tissue autofluorescence. A) Emission spectra of standards with LED excitation spectra overlaid. B) Emission spectra of purified proteins samples. Emission spectra of C) ocular tissues samples and D) skin wound samples. Tissue samples have significant difference in the intensity above $450 \mathrm{~nm}$ that is complicated by multiple protein compositions. Standards were in $0.1 \mathrm{mM}$ concentration in their respective solvents; proteins in powder/fiber forms; and tissues cut into samples as described in the sample preparation section.

\section{Frequency Domain Phase Shift Results}

Phase shifts of the tested samples shown in Figure $\mathbf{5}$ were consistent with lifetimes of fluorescence standards and distinguished ECM proteins that comprise tissues. As seen in the figure, the phase shift angle rose with frequency as expected, following an inverse tangent profile consistent with equation (7). In general, larger phase shift translates to longer lifetime at a specific frequency. For the fluorescence standards, the phase shifts increased from FAD, FITC, to 9CA, in that order, Figure 5A. For the proteins, collagen I consistently measured smaller phase shifts than collagen III at all frequencies, while elastin measured larger phase shifts than the collagen samples, Figure 5B. For the bovine ocular tissues, the scleral tissue measured much larger phase shifts compared to corneal tissue, Figure $\mathbf{5 C}$. The cornea is 
critical to the optical functions of the eye's lens and its transparency is the result of uniform diameter and arrangement of its collagen fibers $[70,71]$, whose crosslinking could be the cause of the smaller phase shift measurements. For the murine wound tissue, larger phase shifts were measured compared to normal skin tissue, indicative of longer lifetime components, Figure 5D. Compared to the collagen measurements, this difference may be due to the relatively greater amount of collagen III (larger phase shift) in the healing wound compared to normal skin [1]. It should be noted that multiple ECM proteins are present in the tissue samples, and collagen itself has multiple lifetime components. The multiexponential decay model, described later, provided better fitting to extract lifetime information from these tissues. Additionally, the phase shift results showed that frequency band of $40-50 \mathrm{MHz}$ has optimal separation for the protein and tissue samples, especially for skin wounds, where data from 10, 20 , and $50 \mathrm{MHz}$ overlaps and do not contribute to their separation.

A)

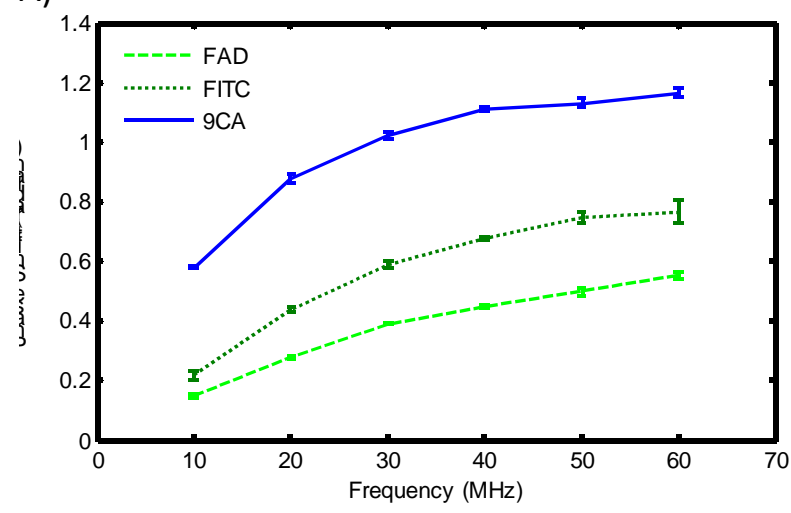

C)

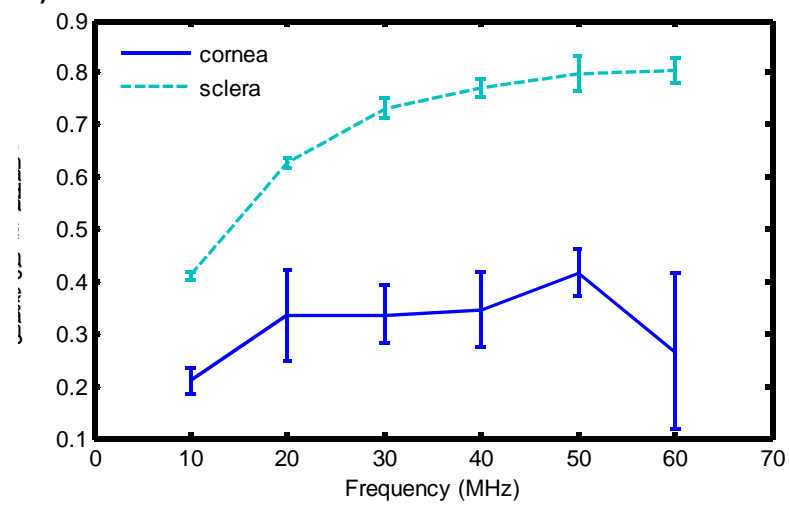

B)

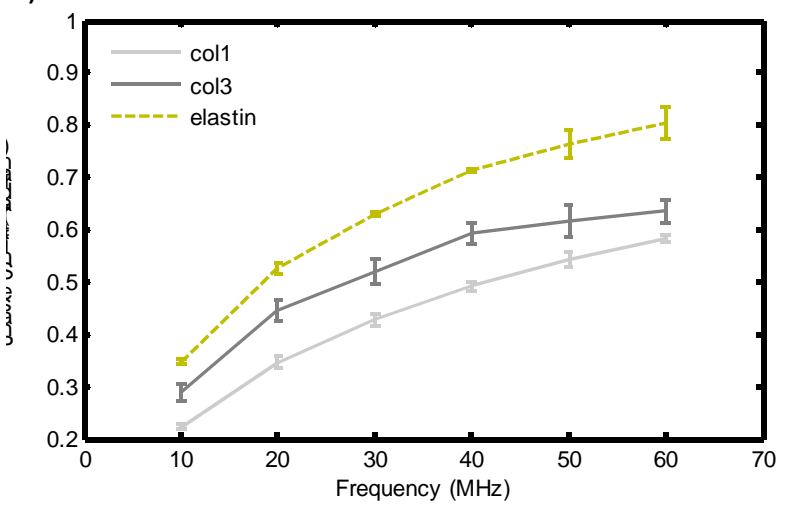

D)

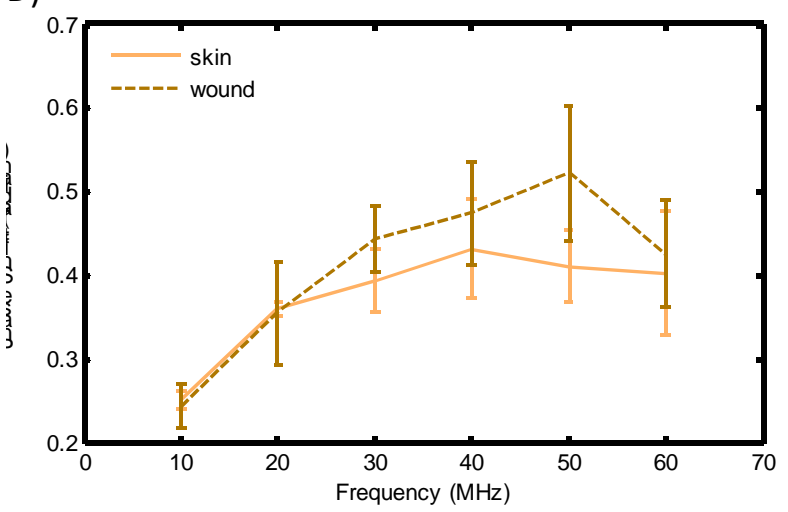

Figure 5. Phase shifts are consistent with lifetimes of fluorescence standards and can distinguish ECM proteins that comprise tissues. A) Fluorescence standards showed increasing phase shift between FAD, FITC, and 9CA, as expected by the order of their increasing lifetimes. B) Phase shift between type I and III collagen are distinct, and type III can be separated from elastin C) Ocular cornea tissues have smaller phase shift than sclera tissue, while D) wound tissues have larger shift compared to normal skin tissue. Error bars denote standard deviations, $\mathrm{n}=3$. 


\section{Frequency Domain Demodulation Results}

Modulation depth ratios of the tested samples indicated that FD demodulation can distinguish collagen proteins that comprise tissues, Figure 6. The modulation depth ratios were seen to drop with frequency as expected, following a decay profile consistent with equation (8). At a particular frequency, deeper demodulation generally translates to longer lifetime. Modulation depth ratios from the standards followed that of the phase shift results, with FAD, FITC, and 9CA in the order of increasing lifetimes, Figure 6A. Again, collagen I versus III measured distinct modulation depth ratios, both shallower than elastin's values, Figure 6B. Ocular tissue demodulation also agreed with the phase results, with sclera having deeper demodulation and thus longer lifetime than cornea tissue, Figure 6C. The murine wound also exhibited deeper demodulation compared to normal skin tissue, Figure 6D, consistent with the aforementioned premise that type III collagen is deposited into wound tissues during healing.

A)

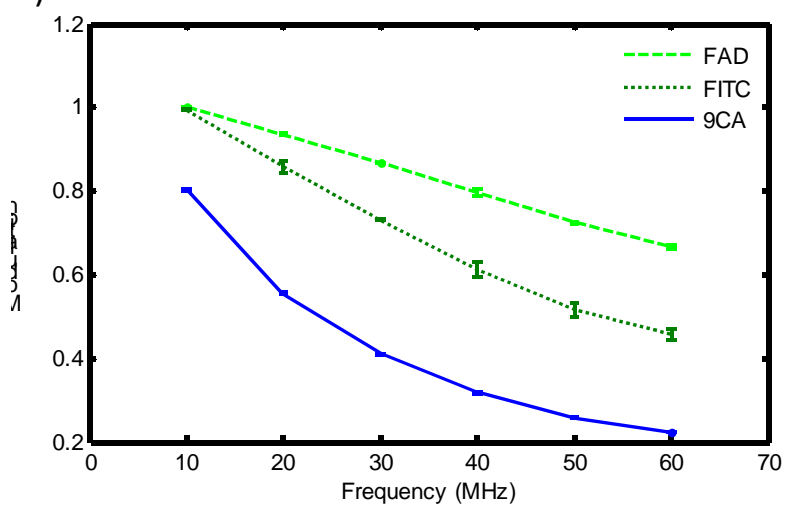

C)

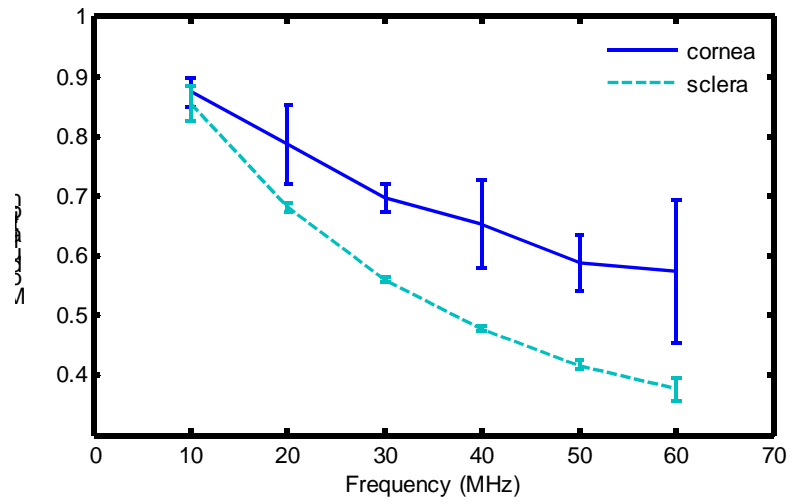

B)

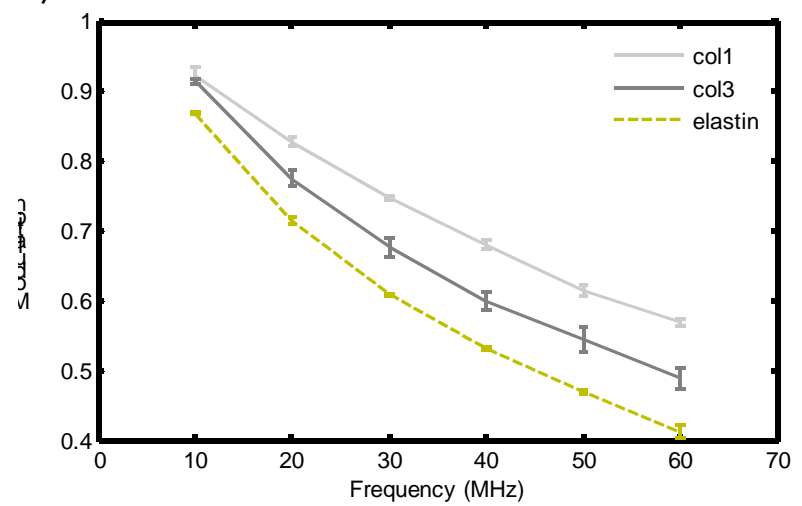

D)



Figure 6. Demodulation results can also distinguish collagen proteins that comprise tissues. A) Fluorescence standards showed deeper demodulations between FAD, FITC, and 9CA, as expected in the order of their increasing lifetimes. B) Modulation depth ratios between type I and III collagen were distinct, and type III could be separated from elastin. C) Ocular cornea tissue had shallower apparent demodulation than sclera tissue, while wound tissue had marginally deeper demodulation compared to normal skin tissue. Error bars denote standard deviations, $n=3$. 
Furthermore, the demodulation results also showed that the frequency band of $40-50 \mathrm{MHz}$ has adequate separation for all samples, especially for ocular and skin wound samples. This indicated that data acquisition in the future can be simplified by focusing on $40-50 \mathrm{MHz}$ bands. In the following section on the multi-exponential model, both the phase and demodulation results were combined in a minimizing function in order to calculate the combined lifetimes.

\section{Multi-exponential Fitting of Collagen, Elastin, and Tissue Lifetimes}

The phase shifts and modulation depth ratios were fitted into single or multi-exponential decay models, weighted with their respective standard deviations, by minimizing equation (9). The fitted lifetimes are shown in Tables 2-5. The lifetime fractional $\alpha$ 's and $\tau^{\prime}$ s are shown along with their apparent $\tau_{\text {avg }}$ for each sample of standards, proteins, and tissues. A single exponential model was used to fit for the fluorescent standards. The fitting showed results of 2.57, 3.94, and 11.75 ns for FAD, FITC, and 9CA, respectively, which are comparable to results in $[58,64,69]$. Protein samples were fitted to a two-exponential model, with a longer lifetime component and a shorter one around $1 \mathrm{~ns}$, consistent with reported values [56, 57, 69]. Collagen III's average lifetime was measured at $5.01 \mathrm{~ns}$, distinct from and longer than the lifetime of collagen I, $3.95 \mathrm{~ns}, \mathrm{p}=0.001$. Other reports have also found longer lifetimes for collagen III compared to I $[51,52]$. This trend for collagen III vs. I has also been confirmed by using a time-domain system,

Supplementary Figure 1. Elastin was distinct from type III collagen, $p=0.0002$, and measured the longest average lifetime of ECM proteins in this study, with a value of $6.78 \mathrm{~ns}$.

Table 2. Single exponential fitting for fluorescence samples

\begin{tabular}{|c|c|c|c|c|c|c|c|c|}
\hline \multirow[t]{2}{*}{ sample } & \multicolumn{2}{|c|}{ lifetimes (ns) } & \multicolumn{2}{|c|}{$\begin{array}{l}\text { pre-exponential } \\
\text { factors }\end{array}$} & \multicolumn{2}{|c|}{$\begin{array}{l}\text { fractional } \\
\text { intensities }\end{array}$} & \multirow{2}{*}{$\begin{array}{c}\begin{array}{c}\text { average } \\
\text { lifetime (ns) }\end{array} \\
\tau_{\text {avg }} \\
\end{array}$} & \multirow[t]{2}{*}{ reference(s) } \\
\hline & $\tau_{1}$ & $\tau_{2}$ & $\alpha_{1}$ & $\alpha_{2}$ & $f_{1}$ & $f_{2}$ & & \\
\hline FAD & $2.57 \pm 0.02$ & - & 1 & - & 1 & - & $2.57 \pm 0.02$ & 69 \\
\hline FITC & $3.94 \pm 0.05$ & - & 1 & - & 1 & - & $3.94 \pm 0.05$ & 58,64 \\
\hline 9CA & $11.75 \pm 0.02$ & - & 1 & - & 1 & - & $11.75 \pm 0.02$ & 69 \\
\hline
\end{tabular}

Table 3. Multi-exponential fitting for protein samples

\begin{tabular}{|c|c|c|c|c|c|c|c|c|}
\hline \multirow[t]{2}{*}{ sample } & \multicolumn{2}{|c|}{ lifetimes (ns) } & \multicolumn{2}{|c|}{$\begin{array}{l}\text { pre-exponential } \\
\text { factors }\end{array}$} & \multicolumn{2}{|c|}{$\begin{array}{l}\text { fractional } \\
\text { intensities }\end{array}$} & \multirow{2}{*}{$\begin{array}{c}\begin{array}{c}\text { average } \\
\text { lifetime (ns) }\end{array} \\
\tau_{\text {avg }} \\
\end{array}$} & \multirow[t]{2}{*}{ reference(s) } \\
\hline & $\tau_{1}$ & $\tau_{2}$ & $\alpha_{1}$ & $\alpha_{2}$ & $f_{1}$ & $f_{2}$ & & \\
\hline col1 & $6.77 \pm 0.24$ & $0.84 \pm 0.04$ & 0.121 & 0.879 & 0.525 & 0.475 & $3.95 \pm 0.13$ & $51,52,56,57,59$ \\
\hline $\mathrm{col} 3$ & $7.46 \pm 0.24$ & $0.76 \pm 0.09$ & 0.150 & 0.850 & 0.633 & 0.367 & $5.01 \pm 0.22$ & $51,52,56,57$ \\
\hline elastin & $9.37 \pm 0.40$ & $1.42 \pm 0.20$ & 0.238 & 0.762 & 0.673 & 0.327 & $6.78 \pm 0.17$ & 56,59 \\
\hline
\end{tabular}


Table 4 Multi-exponential fitting for ocular tissue samples

\begin{tabular}{|c|c|c|c|c|c|c|c|}
\hline \multirow[t]{2}{*}{ sample } & \multicolumn{2}{|c|}{ Lifetimes (ns) } & \multicolumn{2}{|c|}{$\begin{array}{c}\text { Pre-exponential } \\
\text { factors }\end{array}$} & \multicolumn{2}{|c|}{$\begin{array}{l}\text { Fractional } \\
\text { intensities }\end{array}$} & \multirow{2}{*}{$\begin{array}{c}\text { Average lifetime } \\
\text { (ns) }\end{array}$} \\
\hline & $\tau_{1}$ & $\tau_{2}$ & $\alpha_{1}$ & $\alpha_{2}$ & $f_{1}$ & $f_{2}$ & \\
\hline cornea & $8.44 \pm 0.82$ & $0.22 \pm 0.05$ & 0.024 & 0.976 & 0.486 & 0.514 & $4.27 \pm 0.84$ \\
\hline sclera & $9.53 \pm 0.25$ & $1.03 \pm 0.09$ & 0.254 & 0.746 & 0.759 & 0.241 & $7.48 \pm 0.23$ \\
\hline
\end{tabular}

Table 5 Multi-exponential fitting for wound skin samples

\begin{tabular}{|c|c|c|c|c|c|c|c|}
\hline \multirow[t]{2}{*}{ sample } & \multicolumn{2}{|c|}{ Lifetimes (ns) } & \multicolumn{2}{|c|}{$\begin{array}{l}\text { Pre-exponential } \\
\text { factors }\end{array}$} & \multicolumn{2}{|c|}{$\begin{array}{l}\text { Fractional } \\
\text { intensities }\end{array}$} & $\begin{array}{l}\text { Average lifetime } \\
\text { (ns) }\end{array}$ \\
\hline & $\tau_{1}$ & $\tau_{2}$ & $\alpha_{1}$ & $\alpha_{2}$ & $f_{1}$ & $f_{2}$ & $\tau_{\text {avg }}$ \\
\hline skin & $13.08 \pm 0.80$ & $0.72 \pm 0.14$ & 0.049 & 0.951 & 0.484 & 0.516 & $6.72 \pm 0.24$ \\
\hline wound & $18.98 \pm 0.42$ & $1.15 \pm 0.32$ & 0.052 & 0.948 & 0.475 & 0.525 & $9.74 \pm 0.96$ \\
\hline
\end{tabular}

A two-exponential fitting was used for the tissue samples, follow by the calculation of $\tau_{\text {avg }}$. For the ocular tissues, cornea measurements showed much shorter average lifetimes of 4.27 ns than sclera at $7.48 \mathrm{~ns}$. For the murine skin tissues, the $8 \mathrm{~mm}$ murine skin wound at 14 days post-wounding showed a distinct, longer average lifetime at $9.74 \mathrm{~ns}$ versus normal skin at $6.72 \mathrm{~ns}$. This is attributed to the bias of collagen III fibers (with longer lifetimes) laid down during the wound healing process. Murine wounds models used in this study have been reported [1] to express high level of type III collagen around 10-17 days post wounding, coinciding with the day 14 sample in the present study. It should be noted that tissue autofluorescence is comprised of numerous potential fluorophores other than collagen and elastin. The two-exponential fitting, while adequately describing the tissue measurements in this manuscript, could be improved by the addition of spectral-lifetime discrimination, as discussed in the Future Modifications section. 


\section{Performance of FD ECM Detection System}

To provide a comparison of the temporal resolution and the sensitivity of the FD ECM detection system,

Figure 7 shows the standard deviations of repeat lifetime measurements of different sample amounts (collagen I mass and FAD concentration), which correspond to a range of emission intensities (Power). At lower mass/concentrations, FD lifetime measurements remain the same, but include greater standard deviations. A limit of 5\% relative standard deviation was used, i.e. $\sim 20$ times signal to error ratio, as a cut-off for the limit of detection (LOD) determination. The sensitivity results showed that the system has a LOD of $25 \mu \mathrm{g}$ for type I collagen and a LOD of $1 \mu \mathrm{M}$ for FAD detections, while maintaining below $5 \%$ relative standard deviation. At both collagen and FAD LODs, the detected optical power was $\sim 3 \mathrm{nW}$, corresponding to a time resolution of $\sim 200$ ps.

A)

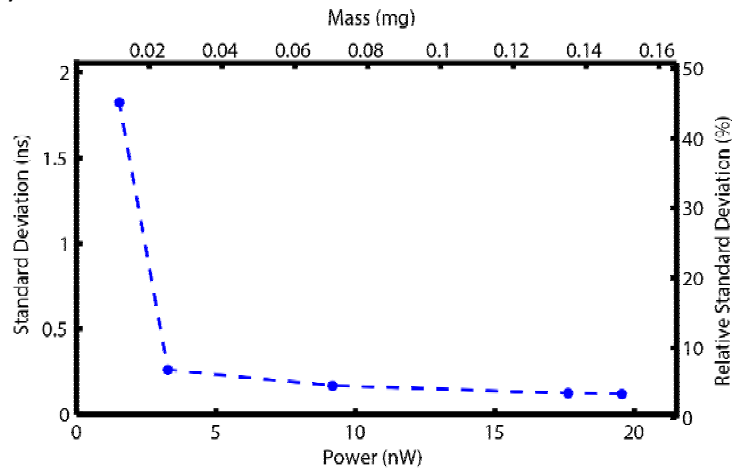

B)

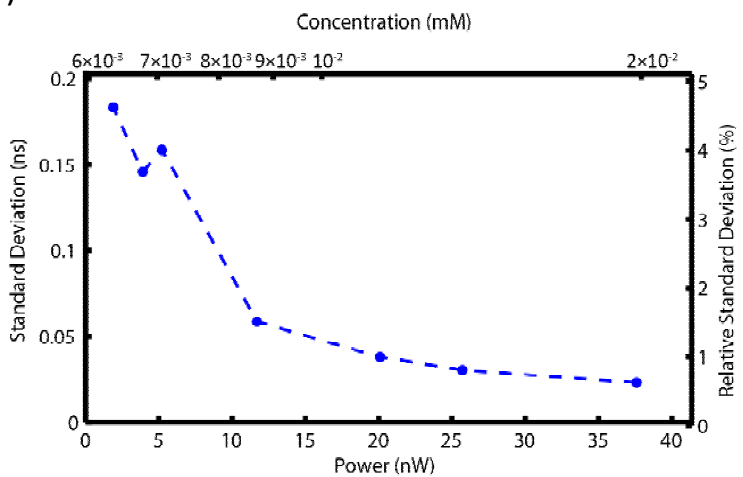

Figure 7. FD ECM detection system sensitivity. A) Limit of detection of solid samples was characterized by using collagen I detections at various masses, with the resultant time resolutions (in ns) and relative standard deviations plotted against power and mass. B) Limit of detection of liquid samples was characterized by using FAD detection at various concentrations, with the resultant time resolution and relative standard deviations plotted against power and concentration.

With respect to skin tissue detections, the probe illuminated a spot size of $5 \mathrm{~mm}$, with penetration depth below $300 \mu \mathrm{m}$ (365 $\mathrm{nm}$ light). This geometry is equivalent to an excitation volume of $6 \mu \mathrm{L}$. Considering skin collagen composition to be $\sim 70 \%$ by weight [72], in vivo measurements would involve $\sim 4.1 \mathrm{mg}$ of collagen using the probe. Therefore, FD ECM detection system provides two orders of magnitude finer sensitivity ( $25 \mu \mathrm{g}$ vs. $4.1 \mathrm{mg}$ ) with 200 picosecond temporal resolution than required for tissue detection, adequate for resolving type I versus type III collagen in the skin. 


\section{Future M odifications for Complex Tissue ECM Collagens}

Although the focus of the detection here is types I and III collagen, ECM compositions of tissue can involve a number of additional collagen types. In ocular tissue, fibrils are primarily composed of type I collagen, although types III, IV, V, VI, VIII, XII and XIII have also been identified to a lesser extent [67, 12]. In skin, collagen IV and VII are associated with basement membrane and dermoepidermal junctions. In addition to multiple collagen types, other ECM proteins like elastin and fibronectin create a complex detection mixture. To resolve these complexities in detection, the current multi-exponential model can benefit from an extra dimension of spectral resolution. First, future modification of the described detection system will include focusing on the frequency band of $40-50 \mathrm{MHz}$, which simplifies data acquisition and analysis without sacrificing collagen and elastin differentiation, as shown by the results given here. Next, instead of integrating all intensities above $400 \mathrm{~nm}$, the emission can be divided into 400-450 nm, 450-500 nm, and 500-550 nm spectral bands using a simple filter wheel setup. These bands may reveal additional biases towards lifetimes of different collagen types and elastin. This should provide improved discrimination in a combined spectral-lifetime technique, as reported by others [73, 74], while still maintaining the simplicity of the LED-based FD ECM detection. Lastly, the multiexponential model could be adapted to predict the fractional contents of major fluorophores. For example, a four exponential model with lifetimes of collagen I, III, elastin defined a priori, and a final residual exponential to account minor fluorophores, can be used to calculate the relative quantity of each defined protein. These future modifications should improve the discrimination of tissue autofluorescence and provide additional quantitative information on protein content without sacrificing system costs.

\section{Conclusion:}

A low cost, low complexity, LED-based FD ECM detection system has been described and shown to be capable of measuring distinct lifetimes for type I and type III collagens and elastin. Fluorescence lifetimes of ECM proteins collagen I, III, and elastin were quantified with 0.2 ns resolution using the low cost frequency domain method, in contrast to the majority of time domain ECM measurements in the literature. The phase shift and modulation of the LED light source were calibrated at each scan 
frequency for both aqueous and solid samples. ECM protein samples were fitted to a two-exponential model, with type I collagen having shorter average lifetimes at 3.95 ns while type III at a longer 5.01 ns, distinct from the elastin lifetime at $6.78 \mathrm{~ns}$. The probe was tested on bovine ocular tissues, with cornea showing much shorter average lifetimes of $4.27 \mathrm{~ns}$ than sclera at $7.48 \mathrm{~ns}$. Furthermore, measurements of $8 \mathrm{~mm}$ murine skin wound at 14 days post-wounding also showed distinct, longer average lifetimes at 9.74 ns versus normal skin at 6.72 ns. Finally, the sensitivity results showed two orders of magnitude lower detection limits than typical tissue and skin collagen compositions, with 200 picosecond lifetime resolutions. Future modification of the FD collagen system will focus on the $40-50 \mathrm{MHz}$ frequency range while separating the emission into three wavelength bands, providing additional spectral-lifetime discriminations. This work specifically targets and optimizes frequency domain lifetime detection of collagen variants in tissue, via a compact, easy-to-use package aimed at enabling clinical wound monitoring, tissue biomechanics monitoring, and other non-invasive biomedical applications. These results and the technique provide additional data points for collagen lifetimes in tissues, and further encourage clinical and interdisciplinary researches to use non-invasive optical biopsy for tissue monitoring. 


\section{Acknowledgements:}

We thank the Center for Wound Repair \& Regeneration at the University of Illinois-Chicago for the animal protocols used in this manuscript. The project was funded by the University of Michigan's research seed fund (J.F.L.). Qiyin Fang was supported by the Natural Science and Engineering Council (NSERC) of Canada. This work was also partly funded by The National Institutes of Health under Grant R01GM50875 (L.C.), and the National Science Foundation under Grant CMMI 1130275 (A.A.). Any opinions, findings, and conclusions or recommendations expressed in this material are those of the authors and do not necessarily reflect the views of the National Science Foundation. These supports are gratefully acknowledged. 


\section{Vitae:}

Rui Liu received his B.S. degree in Mechanical Engineering from Xi'an Jiaotong University, Xi'an, Shaanxi, China in 2013. He is currently pursuing the M.S. degree in the Department of Mechanical Engineering, University of Michigan-Dearborn. His research interests are applied mechanics and biophotonics.

Zhengtuo Zhao received his B.S. degree in Thermal Engineering from Xi'an Jiaotong University, Xi'an, Shaanxi, China in 2013. He is currently pursuing the M.S. degree in the Department of Mechanical Engineering, University of Michigan-Dearborn. His research interests are microfluidics and biophotonics.

Luwei Zou will receive the bachelor's degree in Mechanical Engineering from Xi'an Jiaotong University, Shaanxi, China. She is currently pursuing the master degree in the Department of Mechanical Engineering, University of Michigan-Dearborn. Her research interests are applied mechanics and biophotonics.

Qiyin Fang is an Associate Professor in the Department of Engineering Physics, McMaster University, Hamilton, Ontario. He holds the Canada Research Chair in Biophotonics. His current research interests include: multimodality optical biopsy techniques for real-time clinical diagnosis and guided therapy, optical endoscope designs for gastrointestinal (GI) cancer screening, photodynamic therapy (PDT) photosensitizer uptake and dosimetry, fluorescence lifetime imaging microscopy (FLIM) technologies for high content screening; miniaturized optical sensors for water quality monitoring, and optical sensing and imaging technologies for smart home and aging research.

Lin Chen is a Research Assistant Professor at the Center for Wound Healing and Tissue Regeneration, University of Illinois-Chicago. He received his MD from Chongqing Medical University, China and PhD from Yamagata University, Japan. Dr. Chen's research interests are angiogenesis and innate immunity including interactions between inflammatory cells, keratinocytes and extra-cellular matrix with fibroblasts in cutaneous and mucosal wound healing. Dr. Chen's current research is supported by National Institute of Health and Department of Defense.

Alan Argento is a Professor of Mechanical Engineering at the University of Michigan-Dearborn. He received his Ph.D. in Applied Mechanics from the University of Michigan in 1989. His research interests 
include ocular biomechanics, rate dependence of materials, biological and plant-based materials, impact, structural dynamics, and Elastic Wave Propagation.

Joe Fujiou Lo is an Assistant Professor of Mechanical Engineering, University of Michigan-Dearborn. He received his B.S. in Bioengineering from University of California-Berkeley (2000) and Ph.D. in Biomedical Engineering from University of Southern California (2007). His postdoctoral experience includes ETH Zürich (2008) and University of Illinois-Chicago (2012) with expertise in Microfluidic and Optical Microelectromechanical Systems for Biophotonics applications. The theme of Dr. Lo's research is evolving traditional microengineering towards novel, multifaceted approaches to solve critical biomedical applications. 


\section{References:}

1. J.R. Merkel, B.R. DiPaolo, G.G. Hallock, D.C. Rice. Type I and type III collagen content of healing wounds in fetal and adult rats. Proceedings of the Society of Experimental Biology and Medicine 1988;187:493-497.

2. P.G. Watson, R. Young. Scleral structure, organization, and disease. A review. Experimental Eye Research 2004;78:609-623.

3. R. Beuerman, A. Aplkama, J. Reynaud, N. Nguyen, H. Kikuchi, R. Kalia. Three-dimensional structure of the laminar cribrosa of the living eye. Proceedings of SPIE 2001;4431. doi:10.1117/12.447402.

4. I.A. Darby, T.D. Hewitson. Fibroblast differentiation in wound healing and fibrosis. International Review of Cytology 2007;257:143-179.

5. H.P. Ehrlich. The role of connective tissue matrix in wound healing. Prog. Clin. Biol. Res. $1988 ; 266: 243-258$.

6. M.J. Ranzer, L. Chen, L.A. DiPietro. Fibroblast function and wound breaking strength are impaired by acute ethanol intoxication. Alcohol Clin. Exp. Res. 2011;35:83-90.

7. J.F. Almine, S.G. Wise, A.S. Weiss. Elastin signaling in wound repair. Birth Defects Res. C. Embryo Today 2012;96(3):248-257.

8. M.J.A. Girard, A. Dahlmann-Noor, S. Rayapureddi, J.A. Bechara, B.M.E. Bertin, H. Jones, J. Albon, P.T. Khaw, C.R. Ethier. Quantitative mapping of scleral fiber orientation in normal rat eyes. Investigative Ophthalmology \& Visual Science 2011;52(13):9684-9693.

9. T. Ihanamäki, L.J. Pelliniemi, E. Vuorio. Collagens and collagen-related matrix components in the human and mouse eye. Progress in Retinal and Eye Research 2004;23(4):403-434.

10. J.K. Pijanka, B. Coudrillier, K. Ziegler, T. Sorensen, K.M. Meek, T.D. Nguyen, H.A. Quigley, C. Boote. Quantitative mapping of collagen fiber orientation in non-glaucoma and glaucoma posterior human sclerae. Investigative Ophthalmology \& Visual Science 2012;53(9):5258-5270.

11. R.A. Moses, J.W.J. Grodzki, B.C. Starcher, M.J. Galione. Elastin content of the scleral spur, trabecular mesh, and sclera. Investigative Ophthalmology \& Visual Science 1978;17(8):817-818. 
12. J.A. Rada, V.R. Achen, C.A. Perry, P.W. Fox. Proteoglycans in the human sclera. Evidence for the presence of aggrecan. Investigative Ophthalmology \& Visual Science 1997;38(9):1740-1751.

13. Y. Komai, T. Ushiki. The three-dimensional organization of collagen fibrils in the human cornea and sclera. Investigative Ophthalmology \& Visual Science 1991;32(8):2244-2258.

14. A. Thale, B. Tillmann, R. Rochels. Scanning electron-microscopic studies of the collagen architecture of the human sclera--normal and pathological findings. Ophthalmologica. 1996;210(3):137.

15. O. Schmut. The identification of type III collagen in calf and bovine cornea and sclera. Experimental Eye Research 1977;25(5):505-509.

16. R.E. Lee, P.F. Davison. The collagens of the developing bovine cornea. Experimental Eye Research 1984;39(5):639-652.

17. N.G. Strouthidis, M.J.A. Girard. Altering the way the optic nerve head responds to intraocular pressure-a potential approach to glaucoma therapy. Current Opinion in Pharmacology 2013;13(1):83-89.

18. C.F. Burgoyne, D.J. Crawford, A.J. Bellezza, S.J.K. Francis, R.T. Hart. The optic nerve head as a biomechanical structure: a new paradigm for understanding the role of IOP-related stress and strain in the pathophysiology of glaucomatous optic nerve head damage. Progress in Retinal and Eye Research 2005;24(1):39-73.

19. E.J. Cohen, J.S. Myers. Keratoconus and normal-tension glaucoma: a study of the possible association with abnormal biomechanical properties as measured by corneal hysteresis. Cornea. 2010;29(9):955.

20. K. Trier. The Sclera. Advances in Organ Biology. Vol 10. Philadelphia: Elsevier BV; 2005:353-373.

21. S. Katsuda, Y. Okada, T. Minamoto, Y. Oda, Y. Matsui, I. Nakanishi. Collagen in human atherosclerosis: immunohistomchemical analysis using collagen types-specific antibodies. Arteriosclerosis and Thromobosis 1992;12:494-502.

22. D.A. Newsome, J.M. Foidart, J.R. Hassell, J.H. Krachmer, M.M. Rodrigues, S.I. Katz. Detection of psectific collagen types in normal and keratoconus corneas. Invest. Ophthalmol Vis. Sci. 1981;20(6):738-750. 
23. L.C.U. Junquiera, G. Bignolas, R.R. Brentani. Picrosirius staining plus polarization microscopy, a specific method for collagen detection in tissue sections. Histochemical Journal 1979;11:447-455.

24. L. Chen, M.E. Schrementi, M.J. Ranzer, T.A. Wilgus, L.A. DiPietro. Blockage of mast cell activation reduces cutaneous scar formation. PLOS ONE 2014. DOI: 10.1371/journal.pone.0085226.

25. M.J. Koehler, K. König, P. Elsner, R. Bückle, M. Kaatz. In vivo assessment of human skin aging by multiphoton laser scanning tomography. Optics Letters 2006;31(19):2879-2881.

26. W.R. Zipfel, R.M. Williams, R. Christie, A.Y. Nikitin, B.T. Hyman, W.W. Webb. Live tissue intrinsic emission microscopy using multiphoton-excited native fluorescence and second harmonic generation. PNAS 2003;100(12):7075-7080.

27. Y. Zhang, M.L. Akins, K. Murari, J. Xi, M. Li, K. Luby-Phelps, M. Mahendroo, X. Li. A compact fiber-optic SHG scanning endomicroscope and its application to visualize cervical remodeling during pregnancy. PNAS 2012;109(32):12878-12883.

28. Z. Deyl, K. Macek, M. Adam, O. Vancíková. Studies on the chemical nature of elastin fluorescence. Biochim. Biophys. Acta. 1980;625(2):248-254.

29. D.R. Eyre, M.A. Paz, P.M. Gallop. Cross-linking in collagen and elastin. Annual Review of Biochemistry 1984;53:717-748.

30. E. Fujimori. Changes induced by ozone and ultraviolet light in type I collagen: Bovine Achilles tendon collagen versus rat tail tendon collagen. Eur. J. Biochem. 1985;152:299-306.

31. O. Vančíková, Z. Deyl. Disappearance of tyrosine residues in collagen with age. Suggestion of a possible reaction mechanism. Experimental Gerontology 1974;9(3):123-130.

32. I. Miksík, Z. Deyl. Change in the amount of epsilon-hexosyllysine, UV absorbance, and fluorescence of collagen with age in different animal species. J. Gerontol. 1991;46(3):B111-1116.

33. V.M. Monnier, R.R. Kohn, A. Cerami. Accelerated age-related browning of human collagen in diabetes mellitus. PNAS 1984;81(2):583-587.

34. R. Meerwaldt, T. Links, R. Graaff, S.R. Thorpe, J.W. Baynes, J. Hartog, R. Gans, A. Smit. Simple noninvasive measurement of skin autofluorescence. Ann. N. Y. Acad. Sci. 2005;1043:290-298. 
35. N. Kollias. In vivo fluorescence spectroscopy of nonmelanoma skin cancer. Photochemistry and Photobiology 2001;73(2):178-183.

36. P. Yang, W. Wang, G. Tang. R.R. Alfano. Changes of collagen and nicotinamide adenine dinucleotide in human cancerous and normal prostate tissues studied using native fluorescence spectroscopy with selective excitation wavelength. J. Biomed. Opt. 2010;15(4):047008. doi:10.1117/1.3463479.

37. S. Andersson-Engels, J. Johansson, K. Svanberg, S. Svanberg. Fluorescence imaging and point measurements of tissue: applications to the demarcation of malignant tumors and atherosclerotic lesions from normal tissue. Photochemistry and Photobiology 1991;53(6):807814.

38. P.J. Tadrous, J. Siegel, P.M.W. French, S. Shousha, E. Lalani, G.W.H. Stamp. Fluorescence lifetime imaging of unstained tissues: early results in human breast cancer. The Journal of Pathology 2003;199(3):309-317.

39. V. Lutz, M. Sattler, S. Gallinat, H. Wenck, R. Poertner, F. Fischer. Characterization of fibrillar collagen types using multi-dimensional multiphoton laser scanning microscopy. International Journal of Cosmetic Science 2012;34:209-215.

40. E. Kable, A. Jones, I. Fraser, F. Manconi, M.D. Gorrell. 3-Dimensional imaging of collagen using second harmonic generation Guy Coxa. Journal of Structural Biology 2003;141(1):53-62.

41. A. Zoumi, A. Yeh, B.J. Tromberg. Imaging cells and extracellular matrix in vivo by using secondharmonic generation and two-photon excited fluorescence. PNAS 2002;99(17):11014-11019. doi: $10.1073 /$ pnas.172368799.

42. E. Brown, T. McKee, E. diTomaso, A. Pluen, B. Seed, Y. Boucher, R.K. Jain. Dynamic imaging of collagen and its modulation in tumors in vivo using second-harmonic generation. Nature Medicine 2003;9(6):796-800.

43. K. König, K. Schenke-Layland, I. Riemann, U.A. Stock. Multiphoton autofluorescence imaging of intratissue elastic fibers. Biomaterials 2005;26(5):495-500.

44. L. Brancaleon, A.J. Durkin, J.H. Tu, G. Menaker, J.D. Fallon, S. Lin, S. Jee, J. Chan, R. Wu, W. Lo, H. Tan, W. Lin, J. Chen, T. Young, C. Hsu, C. Dong. Monitoring photoaging by use of multiphoton 
fluorescence and second harmonic generation microscopy. Proc. SPIE 2006;6078. doi:10.1117/12.647009.

45. J. Adur, L. DSouza-Li, M.V. Pedroni, C.E. Steiner, V.B. Pelegati, A.A. de Thomaz, H.F. Carvalho, C.L. Cesar. The severity of Osteogenesis imperfecta and type I collagen pattern in human skin as determined by nonlinear microscopy: proof of principle of a diagnostic method. PLoS One 2013;8(7):e69186. doi: 10.1371/journal.pone.0069186.

46. S. Teng, H. Tan, J. Peng, H. Lin, K.H. Kim, W. Lo, Y. Sun, W. Lin, S. Lin, S. Jee, P.T.C. So, C. Dong. Multiphoton autofluorescence and second-harmonic generation imaging of the ex vivo porcine eye. Invest. Ophthalmol. Vis. Sci. 2006;47(3):1216-1224.

47. P. Matteini, F. Ratto, F. Rossi, R. Cicchi, C. Stringari, D. Kapsokalyvas, F.S. Pavone, R. Pini. Photothermally-induced disordered patterns of corneal collagen revealed by SHG imaging. Optics Express 2009;17(6):4868-4878.

48. P. Steven, M. Müller, N. Koop, C. Rose, G. Hüttmann. Comparison of cornea module and dermalnspect for noninvasive imaging of ocular surface pathologies. J. Biomed. Opt. 2009;14(6):064040. doi: 10.1117/1.3275475.

49. P. Steven, M. Hovakimyan, R.F. Guthoff, G. Hüttmann, O. Stachs. Imaging corneal crosslinking by autofluorescence 2-photon microscopy, second harmonic generation, and fluorescence lifetime measurements. J. Cataract Refract. Surg. 2010;36(12):2150-2159. doi: 10.1016/j.jcrs.2010.06.068.

50. G. Deka, W. Wu, F. Kao. In vivo wound healing diagnosis with second harmonic and fluorescence lifetime imaging. J. Biomed. Opt. 2013;18(6):061222.

51. P. Ashjian, A. Elbarbary, P. Zuk, D.A. Deugarte, P. Benhaim, L. Marcu, M.H. Hedrick. Noninvasive in situ evaluation of osteogenic differentiation by time-resolved laser-induced fluorescence spectroscopy. Tissue Engineering 2004;10(3-4):411-420.

52. L. Marcu, D. Cohen, J.M.I. Maarek, W.S. Grundfest. Characterization of type I, II, III, IV, and V collagens by time-resolved laser-induced fluorescence spectroscopy. Proc. SPIE 2000;3917. doi:10.1117/12.382720. 
53. J.R. Lakowicz. Principle of Fluorescence Spectroscopy, third ed. Springer Science \& Business Media, 2006.

54. D. Elson, J. Requejo-Isidro, I. Munro, F. Reavell, J. Siegel, K. Suhling, P. Tadrous, R. Benninger, P. Lanigan, J. McGinty, C. Talbot, B. Treanor, S. Webb, A. Sandison, A. Wallace, D. Davis, J. Lever, M. Neil, D. Phillips, G. Stamp, P. French. Time-domain fluorescence lifetime imaging applied to biological tissue. Photochem. Photobiol. Sci. 2004;3:795-801.

55. K. Dowling, M.J. Dayel, M.J. Lever, P.M. W. French, J.D. Hares, A.K.L. Dymoke-Bradshaw. Fluorescence lifetime imaging with picosecond resolution for biomedical applications. Optics Letters 1998;23(10):810-812.

56. M.Y. Berezin, S. Achilefu. Fluorescence lifetime measurements and biological imaging. Chem. Rev. 2010;110(5):2641-2684.

57. J.E. Phipps, Y. Sun, M.C. Fishbein, L. Marcu. A fluorescence lifetime imaging classification method to investigate the collagen to lipid ratio in fibrous caps of atherosclerotic plaque. Lasers Surg. Med. 2012;44(7):564-571. doi: 10.1002/Ism.22059.

58. Y. Yuan, J. Hwang, M. Krishnamoorthy, K. Ye, Y. Zhang, J. Ning, R. C. Wang, M. J. Deen, Q. Fang. High-throughput acousto-optic-tunable-filter-based time-resolved fluorescence spectrometer for optical biopsy. Optics Letters 2009;34(7):1132-1134.

59. J.M.I. Maarek, L. Marcu, W.J. Snyder, W.S. Grundfest. Time-resolved fluorescence spectra of arterial fluorescent compounds: reconstruction with the Laguerre expansion technique. Photochem. Photobiol. 2000;71(2):178-187.

60. M.S. Patterson, J.D. Moulton, B.C. Wilson, K.W. Berndt, J.R. Lakowicz. Frequency-domain reflectance for the determination of the scattering and absorption properties of tissue. Applied Optics 1991;30(31):4474-4476.

61. J. Mizeret, G. Wagnières, T. Stepinac, H. Van Den Bergh. Endoscopic tissue characterization by frequency-domain fluorescence lifetime imaging (FD-FLIM). Lasers in Medical Science 1997;12(3):209-217. 
62. A.D. Elder, J.H. Frank, J. Swartling, X. Dai, C.F. Kaminski. Calibration of a wide-field frequencydomain fluorescence lifetime microscopy system using light emitting diodes as light source. J. of Microscopy 2006,224:166-180.

63. B. Yuan, S.R. McClellan, B.F. Al-Mifgai, E.A. Growney, O.A. Komolafe. A cost-efficient frequency domain fluorescence lifetime measurement System. Am. J. Phys. 2010;78(1):28-34.

64. W.J. O’Hagan, M. McKenna, D.C. Sherrington, O.J. Rolinkski, D.J.S. Birch. MHz LED source for nanosecond fluorescence sensing, Meas. Sci. Technology 2002;13:84-91.

65. S. Fantini, B. Barbieri, M.A. Franceschini, E. Gratton. Frequency-domain spectroscopy, in: E. Kohen, J.G. Hirschberg, Applications of Optical Engineering to the Study of Cellular Pathology, Volume 2. Research Signpost, 1999, pp57-66.

66. E. Gratton, M. Limkeman. A continuously variable frequency cross-correlation phase fluorometry with picosecond resolution. Biophys. J. 1983;44:315-324.

67. P.G. Watson, B. Hazleman, P. McCluskey, C. Pavesio. The sclera and systemic disorders. 3rd ed. London: JP Medical, Ltd; 2012.

68. J.R. Lakowizc, G. Laczko, H. Cherek, E. Gratton, M. Limkeman. Analysis of fluorescence decay kinetics from variable-frequency phase shift and modulation data. Biophys. J. 1984;46:463-477.

69. Q. Fang, T. Papaioannou, J.A. Jo, R. Vaitha, K. Shastry, L. Marcu. Time-domain laser-induced fluorescence spectroscopy apparatus for clinical diagnostics. Review of Scientific Instruments 2004;75(1):151-162.

70. T. Ihanamaki, L.J. Pelliniemi, E. Vuorio. Collagen and collagen-related matrix components in the human and mouse eye. Progress in Retinal and Eye Research 2004;23: 403-434.

71. G.E. Marshall, A.G.P. Konstas, W.R. Lee. Collagen in ocular tissues. British Journal of Ophthalmology 1993;77:515-524.

72. R.E. Neuman, M.A. Logan. The determination of collagen and elastin in tissues. Journal of Biological Chemistry 1950;186:549-556.

73. H. Xie, J. Bec, J. Liu, Y. Sun, M. Lam, D.R. Yankelevich, L. Marcu. Multispectral scanning timeresolved fluorescence spectroscopy (TRFS) technique for intravascular diagnosis. Biomedical Optics Express 2012;3(7):1521-1533. 
74. D. Chorvat Jr., A. Chorvatova. Multi-wavelength fluorescence lifetime spectroscopy: a new approach to the study of endogenous fluorescence in living cells and tissues. Laser Physics Letters 2009;6(3):175-193. 
Table 1. System Components:

\begin{tabular}{l|l|l}
\hline Component & Relevant Spec & Price \\
\hline Thorlabs Modulated LED Source & $700 \mathrm{~mA}, 10-90 \mathrm{MHz}$ sinusoid & $\$ 2510$ \\
1" Lens tube components & SM1 lens tube, SM1 collimator & $\$ 450$ \\
SM1 optics & $1 "$ lens and lens tubes & $\$ 200$ \\
Colored Glass Bandpass & $275-375 \mathrm{~nm}$ & $\$ 86$ \\
Stellarnet 7 around 1 probe & $600 \mu \mathrm{m}$, solarization resistant & $\$ 600$ \\
Manual filter wheel & 6 position, 1" optics & $\$ 145$ \\
Thorlabs Longpass FEL0400 & $400 \mathrm{~nm}$ & $\$ 73(2)$ \\
Thorlabs APD120A2 Photodiode & $\mathrm{DC}-50 \mathrm{MHz}(3 \mathrm{~dB}), 2.5 \mathrm{e} 6 \mathrm{~V} / \mathrm{W}$ & $\$ 1070$ \\
Rigol DS1000B Oscilloscope & $200 \mathrm{MHz}, 2 \mathrm{GSa} / \mathrm{s}$ & $\$ 945$ \\
\hline
\end{tabular}

Table 2. Single exponential fitting for fluorescence samples

\begin{tabular}{|c|c|c|c|c|c|c|c|c|}
\hline \multirow[t]{2}{*}{ sample } & \multicolumn{2}{|c|}{ lifetimes (ns) } & \multicolumn{2}{|c|}{$\begin{array}{l}\text { pre-exponential } \\
\text { factors }\end{array}$} & \multicolumn{2}{|c|}{$\begin{array}{l}\text { fractional } \\
\text { intensities }\end{array}$} & \multirow{2}{*}{$\begin{array}{c}\begin{array}{c}\text { average } \\
\text { lifetime (ns) }\end{array} \\
\tau_{\text {avg }}\end{array}$} & \multirow[t]{2}{*}{ reference(s) } \\
\hline & $\tau_{1}$ & $\tau_{2}$ & $\alpha_{1}$ & $\alpha_{2}$ & $f_{1}$ & $f_{2}$ & & \\
\hline FAD & $2.57 \pm 0.02$ & - & 1 & - & 1 & - & $2.57 \pm 0.02$ & 69 \\
\hline FITC & $3.94 \pm 0.05$ & - & 1 & - & 1 & - & $3.94 \pm 0.05$ & 58,64 \\
\hline 9CA & $11.75 \pm 0.02$ & - & 1 & - & 1 & - & $11.75 \pm 0.02$ & 69 \\
\hline
\end{tabular}

Table 3. Multi-exponential fitting for protein samples

\begin{tabular}{|c|c|c|c|c|c|c|c|c|}
\hline \multirow[t]{2}{*}{ sample } & \multicolumn{2}{|c|}{ lifetimes (ns) } & \multicolumn{2}{|c|}{$\begin{array}{l}\text { pre-exponential } \\
\text { factors }\end{array}$} & \multicolumn{2}{|c|}{$\begin{array}{l}\text { fractional } \\
\text { intensities } \\
\end{array}$} & \multirow{2}{*}{$\begin{array}{c}\begin{array}{c}\text { average } \\
\text { lifetime (ns) }\end{array} \\
\tau_{\text {avg }}\end{array}$} & \multirow[t]{2}{*}{ reference(s) } \\
\hline & $\tau_{1}$ & $\tau_{2}$ & $\alpha_{1}$ & $\alpha_{2}$ & $f_{1}$ & $f_{2}$ & & \\
\hline col1 & $6.77 \pm 0.24$ & $0.84 \pm 0.04$ & 0.121 & 0.879 & 0.525 & 0.475 & $3.95 \pm 0.13$ & $51,52,56,57,59$ \\
\hline $\mathrm{col} 3$ & $7.46 \pm 0.24$ & $0.76 \pm 0.09$ & 0.150 & 0.850 & 0.633 & 0.367 & $5.01 \pm 0.22$ & $51,52,56,57$ \\
\hline elastin & $9.37 \pm 0.40$ & $1.42 \pm 0.20$ & 0.238 & 0.762 & 0.673 & 0.327 & $6.78 \pm 0.17$ & 56,59 \\
\hline
\end{tabular}

Table 4 Multi-exponential fitting for ocular tissue samples

\begin{tabular}{|c|c|c|c|c|c|c|c|}
\hline \multirow{2}{*}{ sample } & \multicolumn{2}{|c|}{ Lifetimes (ns) } & \multicolumn{2}{|c|}{$\begin{array}{c}\text { Pre-exponential } \\
\text { factors }\end{array}$} & \multicolumn{2}{|c|}{$\begin{array}{l}\text { Fractional } \\
\text { intensities }\end{array}$} & \multirow{2}{*}{$\begin{array}{c}\text { Average lifetime } \\
\text { (ns) } \\
\tau_{\text {avg }}\end{array}$} \\
\hline & $\tau_{1}$ & $\tau_{2}$ & $\alpha_{1}$ & $\alpha_{2}$ & $f_{1}$ & $f_{2}$ & \\
\hline cornea & $8.44 \pm 0.82$ & $0.22 \pm 0.05$ & 0.024 & 0.976 & 0.486 & 0.514 & $4.27 \pm 0.84$ \\
\hline sclera & $9.53 \pm 0.25$ & $1.03 \pm 0.09$ & 0.254 & 0.746 & 0.759 & 0.241 & $7.48 \pm 0.23$ \\
\hline
\end{tabular}

Table 5 M ulti-exponential fitting for wound skin samples

\begin{tabular}{|c|c|c|c|c|c|c|c|}
\hline \multirow[t]{2}{*}{ sample } & \multicolumn{2}{|c|}{ Lifetimes (ns) } & \multicolumn{2}{|c|}{$\begin{array}{l}\text { Pre-exponential } \\
\text { factors }\end{array}$} & \multicolumn{2}{|c|}{$\begin{array}{l}\text { Fractional } \\
\text { intensities }\end{array}$} & \multirow{2}{*}{$\begin{array}{c}\text { Average lifetime } \\
\text { (ns) } \\
\tau_{\text {avg }}\end{array}$} \\
\hline & $\tau_{1}$ & $\tau_{2}$ & $\alpha_{1}$ & $\alpha_{2}$ & $\mathrm{f}_{1}$ & $f_{2}$ & \\
\hline skin & $13.08 \pm 0.80$ & $0.72 \pm 0.14$ & 0.049 & 0.951 & 0.484 & 0.516 & $6.72 \pm 0.24$ \\
\hline wound & $18.98 \pm 0.42$ & $1.15 \pm 0.32$ & 0.052 & 0.948 & 0.475 & 0.525 & $9.74 \pm 0.96$ \\
\hline
\end{tabular}




\section{Vitae:}

Rui Liu received his B.S. degree in Mechanical Engineering from Xi'an Jiaotong University, Xi'an, Shaanxi, China in 2013. He is currently pursuing the M.S. degree in the Department of Mechanical Engineering, University of Michigan-Dearborn. His research interests are applied mechanics and biophotonics.

Zhengtuo Zhao received his B.S. degree in Thermal Engineering from Xi'an Jiaotong University, Xi'an, Shaanxi, China in 2013. He is currently pursuing the M.S. degree in the Department of Mechanical Engineering, University of Michigan-Dearborn. His research interests are microfluidics and biophotonics.

Luwei Zou will receive the bachelor's degree in Mechanical Engineering from Xi'an Jiaotong University, Shaanxi, China. She is currently pursuing the master degree in the Department of Mechanical Engineering, University of Michigan-Dearborn. Her research interests are applied mechanics and biophotonics.

Qiyin Fang is an Associate Professor in the Department of Engineering Physics, McMaster University, Hamilton, Ontario. He holds the Canada Research Chair in Biophotonics. His current research interests include: multimodality optical biopsy techniques for real-time clinical diagnosis and guided therapy, optical endoscope designs for gastrointestinal (GI) cancer screening, photodynamic therapy (PDT) photosensitizer uptake and dosimetry, fluorescence lifetime imaging microscopy (FLIM) technologies for high content screening; miniaturized optical sensors for water quality monitoring, and optical sensing and imaging technologies for smart home and aging research.

Lin Chen is a Research Assistant Professor at the Center for Wound Healing and Tissue Regeneration, University of Illinois-Chicago. He received his MD from Chongqing Medical University, China and PhD from Yamagata University, Japan. Dr. Chen's research interests are angiogenesis and innate immunity including interactions between inflammatory cells, keratinocytes and extra-cellular matrix with fibroblasts in cutaneous and mucosal wound healing. Dr. Chen's current research is supported by National Institute of Health and Department of Defense.

Alan Argento is a Professor of Mechanical Engineering at the University of Michigan-Dearborn. He received his Ph.D. in Applied Mechanics from the University of Michigan in 1989. His research interests 
include ocular biomechanics, rate dependence of materials, biological and plant-based materials, impact, structural dynamics, and Elastic Wave Propagation.

Joe Fujiou Lo is an Assistant Professor of Mechanical Engineering, University of Michigan-Dearborn. He received his B.S. in Bioengineering from University of California-Berkeley (2000) and Ph.D. in Biomedical Engineering from University of Southern California (2007). His postdoctoral experience includes ETH Zürich (2008) and University of Illinois-Chicago (2012) with expertise in Microfluidic and Optical Microelectromechanical Systems for Biophotonics applications. The theme of Dr. Lo's research is evolving traditional microengineering towards novel, multifaceted approaches to solve critical biomedical applications. 
Page 37 of 37 